\title{
O MUNDO PERNAMBUCANO ATRAVÉS DE FONTES DE INFORMAÇÃO: 1965-2003
}

\section{EL MUNDO PERNAMBUCANO A TRAVÉS DE FUENTES DE INFORMA- CIÓN: 1965-2003}

\begin{abstract}
Cecília Prysthon - cmfp@ufpe.br
Especialista em Informação Tecnológica pela Universidade Federal de Pernambuco (UFPE). Professora do Departamento de Ciência da Informação da Universidade Federal de Pernambuco (UFPE).

Murilo Artur Araújo da Silveira - muriloas@gmail.com Mestre em Ciência da Informação pela Pontifícia Universidade Católica de Campinas

(PUCCAMP).

\section{Lígia Santos da Silva - ligiadiaspora@hotmail.com}

Bacharel em Biblioteconomia pela Universidade Federal de Pernambuco (UFPE). Bibliotecária da Universidade Federal da Paraíba (UFPB).
\end{abstract}

\footnotetext{
Resumo

$\mathrm{O}$ artigo descreve o estudo feito com fins de compilar uma bibliografia de fontes de informação sobre o assunto Pernambuco, no período de 1965 a 2003. Apresenta resultados parciais do estudo, relativos à quantidade de registros por assunto e localização dos mesmos em seis bibliotecas da Cidade do Recife. O produto final deve incluir: referência bibliográfica, nota de conteúdo e biblioteca, com recuperação através de índices onomástico e de assunto. A bibliografia pretende servir de instrumento de trabaIho e pesquisa, tanto para profissionais da informação, quanto para diferentes tipos de usuários.
}

Palavras-Chave: Fontes de informação. Bibliografias. Pernambuco. 


\section{INTRODUÇÃO}

Em 1964 foi editado, pela Imprensa Universitária do Recife, o livro "Bibliografia de obras de referência pernambucanas", de autoria de Edson Nery da Fonseca (1964), listando 75 itens de diferentes tipos de materiais, com datas de 1837 a 1955. Segundo o autor, a cobertura compreendeu obras de referência publicadas em Pernambuco ou relativas a assuntos e instituições pernambucanas.

Em 1999, Ann Hartness edita, pela Briquet de Lemos/Livros de Brasília, o livro "Brasil: obras de referência - 1965-1998", no qual aparecem 88 registros sobre Pernambuco, incluindo diferentes tipos de materiais, nas áreas de Humanidades, Artes e Ciências Sociais, com variada cobertura de assuntos.

A oferta da disciplina "Fontes de Informação" no Curso de Biblioteconomia da UFPE, favoreceu a execução de atividade pedagógica no segundo semestre de 2002 , com trabalho de atualização da listagem de Hartness (de 1998 a 2003), em bibliotecas selecionadas na cidade do Recife. Tarefa esta que resultou em 129 itens em 10 bibliotecas visitadas pelos alunos, com participação de monitoria da disciplina. $O$ intuito foi envolver os alunos em uma atividade de pesquisa durante seu processo de formação, sem ficar limitada a momentos ou espaços pontuais do curso.
Devido à carência de ferramentas de trabalho, impressos ou eletrônicos, que reúnam fontes de informação, obras de referência ou similar, houve o interesse em prosseguir com a referida atividade. Isto porque se entende que através desse tipo de repertório pode se conhecer determinada região, estado, cultura, história e sua produção para servir de instrumento de estudo e pesquisa para a sociedade. Além disso, convém acrescentar que o levantamento do material foi feito através de consulta local a unidades de informação do Recife, enquanto no levantamento de Hartness (1999) a consulta foi centrada em acervos de sete bibliotecas brasileiras do Rio de Janeiro, São Paulo, Brasília e três bibliotecas americanas.

A organização de bibliografias pode servir de subsídio para análise e dimensão de assuntos e áreas específicas estudadas, ou ainda serem exploradas em futuras investigações. Este tipo de coletânea contribui ainda para mostrar um panorama do controle bibliográfico estadual, suas circunstâncias e adjacências, através de diferentes temáticas, em diferentes períodos de tempo, através de múltiplas formas do conhecimento em abordagens de/sobre Pernambuco.

A importância do tema da pesquisa se situa na concepção de instrumentos (tradicionais, formais e clássicos) a serem utilizados para fins de consulta, estudo e pesquisa para alunos, professores, pesqui- 
sadores, técnicos e usuários em geral, bem como instituições de ensino, setor público e empresarial.

Desse modo, a obra de Ann Hartness (1999) foi fonte de inspiração e ponto de partida para a elaboração e execução do projeto. A pesquisa foi retomada em 2003 e tem previsão para término em 2008. Intitulada "O mundo pernambucano através de fontes de informação", o projeto visa colher documentos de/sobre Pernambuco nas áreas de Artes, Humanidades e Ciências Sociais, com ampla cobertura de assuntos, no período de 1965 a 2003.

Tem como objetivos:

- Resgatar a produção relativa a assuntos de/sobre Pernambuco no período;

- Compilar e analisar materiais de referências pertinentes a Pernambuco, em bibliotecas públicas, universitárias e especializadas do Recife; e

- Ordenar os registros sob representação dos assuntos, com descrição, resumo e localização física dos documentos.

\section{REVISÃO DA LITERATURA}

O tema da pesquisa se insere no contexto da bibliografia, que por sua vez está agrupada na tipologia de obra de referência, ao se falar da composição e variedade da coleção do acervo de bibliotecas, uni- dades de informação, centros de documentação e de informação.

A obra de Grogan (1995) menciona obra de referência como "aquela destinada, em virtude de organização e tratamento de dados e seu conteúdo temático, a ser consultada em busca de informações específicas e não para ser lida inteiramente", conforme a American Library Association (ALA) - Glossary of Library and Information Science.

Por sua vez, Cunha (2001) aponta que as bibliografias, em clássica divisão, são consideradas documentos terciários que têm como função principal ajudar o leitor na pesquisa de fontes primárias e secundárias, sendo que na maioria não trazem nenhum conhecimento do assunto como um todo. Isto é, são sinalizadores de localização ou indicadores de documentos primários ou secundários, além de informação factual. O mesmo autor, de forma mais explícita, afirma que bibliografia consiste em uma "lista de referências bibliográficas relativas aos diversos tipos de fontes de informação sobre determinado assunto ou pessoa", sendo geralmente organizada em ordem alfabética de autores. Em termos de cobertura, pode ser exaustiva ou seletiva, podendo trazer apenas a referência ou incluir anotações sobre o item analisado.

Para Figueiredo e Cunha (1967) a função da bibliografia consiste em fornecer dados relativos à produção bibliográfica de 
um país, estado, região, e informar sobre a atividade intelectual em várias áreas do conhecimento. São obras de pesquisa, consulta, indicando o que já foi realizado nos domínios do saber e visam facilitar o trabalho científico, técnico e cultural.

Com mais detalhe e amplitude Fonseca (1964) afirma que as bibliografias assumem a função instrumental de oferecer um panorama da produção editorial e bibliográfica de locais, instituições, personalidades, grupos de pessoas e assuntos, e de desvendar as nuances, características e peculiaridades do repertório cultural (aspectos históricos, econômicos e sociais) e científico produzido por uma região/nação.

Mais recentemente, usando a expressão guias bibliográficos, Bufrem (1993, p. 5 ) afirma que:

[...] são valiosos instrumentos para sistemas de informação, pois além de facilitarem as buscas dos usuários, contribuem para formar imagens da produção bibliográfica em determinada área do conhecimento, num período de tempo, num certo local e/ou distribuição num conjunto de bibliotecas.

Sem fugir, necessariamente, às divisões clássicas e tradicionais da área, surge na última década a expressão fontes de informação, englobando a variedade de obras de referência e indo até além. Com esta concepção se posiciona Hartness (1999) ao entender por fonte de informação qualquer documento que forneça uma informação específica, constituindo-se de elementos fundamentais para responder uma consulta, buscar e preencher uma necessidade de informação. Em determinados momentos e situações, essas fontes podem se caracterizar como obras ou fontes de referência por possuírem informações específicas, mesmo sem terem sido concebidas com tal.

Assim, as bibliografias podem ser entendidas como os guias de fontes de informações que assinalam temas, pessoas, instituições, locais, identificando seus dados descritivos e temáticos, localizando-os fisicamente, através de arranjo alfabético, cronológico, temático, entre outros, concomitantemente ou não.

As bibliografias podem ser exaustivas, seletivas, sinaléticas, analíticas, críticas, retrospectivas e correntes (NORO$\mathrm{NHA}, 1982)$. Estas categorias estão relacionadas com a sua natureza, sendo que os tipos mais encontrados nos últimos anos são as analíticas e as retrospectivas.

Os guias bibliográficos analíticos se caracterizam por apresentar, além da referência bibliográfica, resumos, notas e/ou análises do conteúdo do documento descrito. Os retrospectivos registram os documentos relacionados a um período já ultrapassado. As descrições do conteúdo documental fornecem elementos para que os usuários tomem decisões de acordo com suas necessidades. A cobertura do período estabelece parâmetros para a pesquisa e 
oferece itens bibliográficos num espaço de tempo determinado.

Para exemplificar estes dois casos, evidencia-se a obra de Ann Hartness (1999), que serviu de modelo para o projeto. No livro, os itens estão arranjados em ordem temática, com subdivisões de forma e/ou geográfica, trazendo referências bibliográficas organizadas alfabeticamente $\mathrm{e}$ notas de conteúdo, referentes aos documentos editados de 1965 a 1998.

Sobre a importância do documento bibliografia, Cunha (2001) defende que o uso regular e efetivo de fontes apropriadas, impressas/formais é a chave para se alcançar sucesso na pesquisa, desenvolvimento e conhecimento da produção de diferentes áreas do conhecimento, assim como atividades ligadas à ciência e tecnologia. $\mathrm{O}$ autor ainda afirma que o praticante de ciência e tecnologia que souber utilizar as fontes existentes, e que tenha paciência, energia, perspicácia, terá vantagem sobre os que não possuam as habilidades necessárias ou que não saibam utilizá-las.

$\mathrm{Na}$ tentativa de produzir um instrumento de trabalho e de consulta sobre um assunto, vale a pena se reportar à tradicional e clássica idéia de Ranganathan, ao criar cinco leis (1931) sobre a importância do livro. Essas leis, na década de 90, são re-estudadas e ainda discutidas e analisadas em sua modernidade. Conforme explica Figueiredo (1992), para Ranganathan a tuir abordagens subjetivas ou puramente impressionistas, isso quando se refere a métodos como: consultas a "fontes bibliográficas" e a especialistas, quando se refere à avaliação de coleções com abordagem impressionista. Como princípio, o bibliotecário deveria estar treinado e preocupar-se com a exploração de recursos bibliográficos, avaliar coleções e serviços em termos das necessidades do usuário. Por extensão, cada usuário deve poder saber o que existe sobre cada assunto, onde encontrar, buscar o material, e o mesmo estar disponível para atender a sua necessidade.

Finalmente, por que elaborar listagens de documentos, de fontes, enfim, construir uma bibliografia? Talvez Campello, Cendón e Kremer (2000) cheguem a responder essa pergunta quando destacam: a importância da literatura especializada para uma determinada área do conhecimento, a sua identificação, coleta, organização e preservação estão entre as responsabilidades mais importantes do profissional da informação. Ainda Campello, Cendón e Kremer (2000) complementam que, apesar de toda evolução tecnológica - e mesmo por causa dela - a necessidade de se conhecer as fontes e saber identificar e promover o acesso à informação pertinente, continua sendo tão importante quanto sempre foi para os profissionais que se dedicam ao atendimento do usuário. 


\section{METODOLOGIA}

A presente pesquisa segundo seus procedimentos se apresenta como bibliográfica e documental. Constitui-se em técnicas de seleção, análise, tratamento $\mathrm{e}$ organização de obras de referência e fontes de informação de/sobre Pernambuco, para a recuperação temática do conjunto de registros coletados. Busca-se fornecer subsídios para consultas, estudos e pesquisas de estudantes, professores, pesquisadores, instituições e usuários em geral.

As etapas realizadas no desenvolvimento do projeto são expostas, a seguir:

1. análise da literatura sobre bibliografias e fontes de informação;

2. definição e seleção dos parâmetros para a coleta das fontes de informação e das bibliotecas da cidade a serem visitadas;

3. busca e coleta, em catálogos manuais e informatizados, dos materiais pertinentes à pesquisa;

4. descrição dos itens em forma de referência, incluindo notas de conteúdo;

5. indexação e digitação dos itens;

6. compilação das fontes de informações afetas aos objetivos do projeto;
7. arranjo dos registros segundo assuntos e outras categorias;

8. construção dos índices de assunto e de nomes.

Na seleção das Bibliotecas da Cidade do Recife a serem visitadas, foram escolhidas as bibliotecas que possuíssem coleções em conformidade com os propósitos do projeto, isto é, as unidades que tivessem itens bibliográficos de/sobre o Estado de Pernambuco. O total de bibliotecas selecionadas para visitação foram treze, das quais foram destacadas seis, pela representação quantitativa, a saber:

-Biblioteca Pública do Estado de Pernambuco - Coleção Pernambucana (BPE);

-Fundação Joaquim Nabuco (FUNDAJ)

-Instituto Tecnológico do Estado de Pernambuco (ITEP);

-Universidade Católica de Pernambuco (UNICAP);

-Universidade Federal de Pernambuco (UFPE);

-Universidade Federal Rural de Pernambuco (UFRPE);

As demais sete bibliotecas não alcançaram o índice de dez itens do total encontrado.

As buscas, referências e notas de conteúdo dos itens bibliográficos afetos à pesquisa foram realizadas in-loco. As o- 
bras foram localizadas, selecionadas e incluídas através de catálogos manuais e automatizados, sob critérios de assunto e palavra-chave no título. Ainda sob exame de cada obra para verificar a abordagem do conteúdo e preparar a descrição do mesmo.

Para a descrição dos itens, foi escoIhido o formato recomendado pela NBR 6023/2002 da ABNT, referente à elaboração de referências bibliográficas, que normaliza a ordem e a disposição dos elementos descritivos de diversos materiais. As notas de conteúdo estão dispostas, logo após as referências. Estas notas buscam oferecer informações de conteúdo e de características físicas dos materiais. Os julgamentos de valor foram descartados pelo fato da compilação não estar sendo elaborada para um público específico e porque necessidades de informação podem variar. Também são registrados os locais para obtenção dos itens. A seguir, será mostrado um exemplo, conforme descrito.

FERRAZ, Marilourdes. A presença da mulher nas redações. Recife: Instituto Antônio Camelo de Estudos de Comunicação Social, 1999. (Série Grandes Nomes da Comunicação). // Destaca a presença feminina nos jornais pernambucanos, com um total de trinta mulheres que atuaram em jornais, rádios, televisões e agências de publicidade de Pernam- buco. Inclui breve biografia de cada personagem com fotos.

LOCALIZAÇÃO: FUNDAJ; UNICAP

Na etapa de indexação dos itens foram analisados os descritores adotados pelas bibliotecas visitadas, com a finalidade de verificar a pertinência ou não, das palavras-chave utilizadas para representar o conteúdo dos documentos. Esta fase exigiu um grande esforço pelo fato de cada biblioteca possuir sua política de representação descritiva e temática, dificultando, em alguns casos, a escolha dos descritores.

Logo após, todos os documentos coletados foram digitados, com ordenação temática semelhante ao livro de Ann Hartness (1999), com algumas adaptações. Ressalta-se que foi criado um modelo estrutural de documento no Microsoft Word, para que a visualização dos itens depois de impressos fosse a melhor possível. Em seguida, realizou-se a conferência dos títulos encontrados, ordenando todos os itens numa listagem geral. Registra-se ainda que a presente pesquisa está em fase de conclusão, detendo-se à confecção dos índices onomástico e de assunto. Os grupos temáticos adotados pelo estudo em questão são apresentados através do Quadro 1. 


\begin{tabular}{ccc|}
\hline ARRANJO & GRUPOS TEMATICOS & SIGLA \\
\hline Obras de Referência & Bibliografias & BIB \\
& Fontes Gerais & FOG \\
& Arquitetura & ARQ \\
& Arte & ART \\
& Bibliotecas, Arquivos e Museus & BAM \\
& Biografias & BIO \\
& Cinema e Teatro & CIT \\
& Comunicação & COM \\
& Condições Sociais & COS \\
Assuntos & Direito & DIR \\
& Economia e Agricultura & ECA \\
& Educação & EDU \\
& Folclore e Cultura Popular & FCP \\
& Geografia & GEO \\
& Grupos Étnicos & GET \\
& História & HIS \\
& Meio Ambiente & MAM \\
\hline Música & MUS \\
& Política e Governo & POG \\
\hline Religião & REL \\
\hline
\end{tabular}

Quadro 1 - Denominação dos grupos temáticos

\section{RESULTADOS E DISCUSSÃO}

O total de registros localizados, selecionados e examinados chega a 594, distribuídos nos seguintes grupos, conforme mostra o Quadro 1 e o Gráfico 1.

O gráfico 1 retrata áreas de maior e menor incidência da produção de fontes de informação de assuntos relativos ao Estado de Pernambuco nas áreas de humanidades, ciências sociais e artes, incluindo $100 \%$ das fontes coletadas. Ressalta-se que os itens existem e foram localizados nos locais visitados, ou seja, dentro dos limites da pesquisa. Outras produções concernentes ao tema da pesquisa podem estar presentes em outras bibliotecas ou centros de informação não selecionados para participar deste levantamento. Vale acrescentar também que a bibliografia não se caracteriza como exaustiva, mas como bibliografia especializada anotada. 


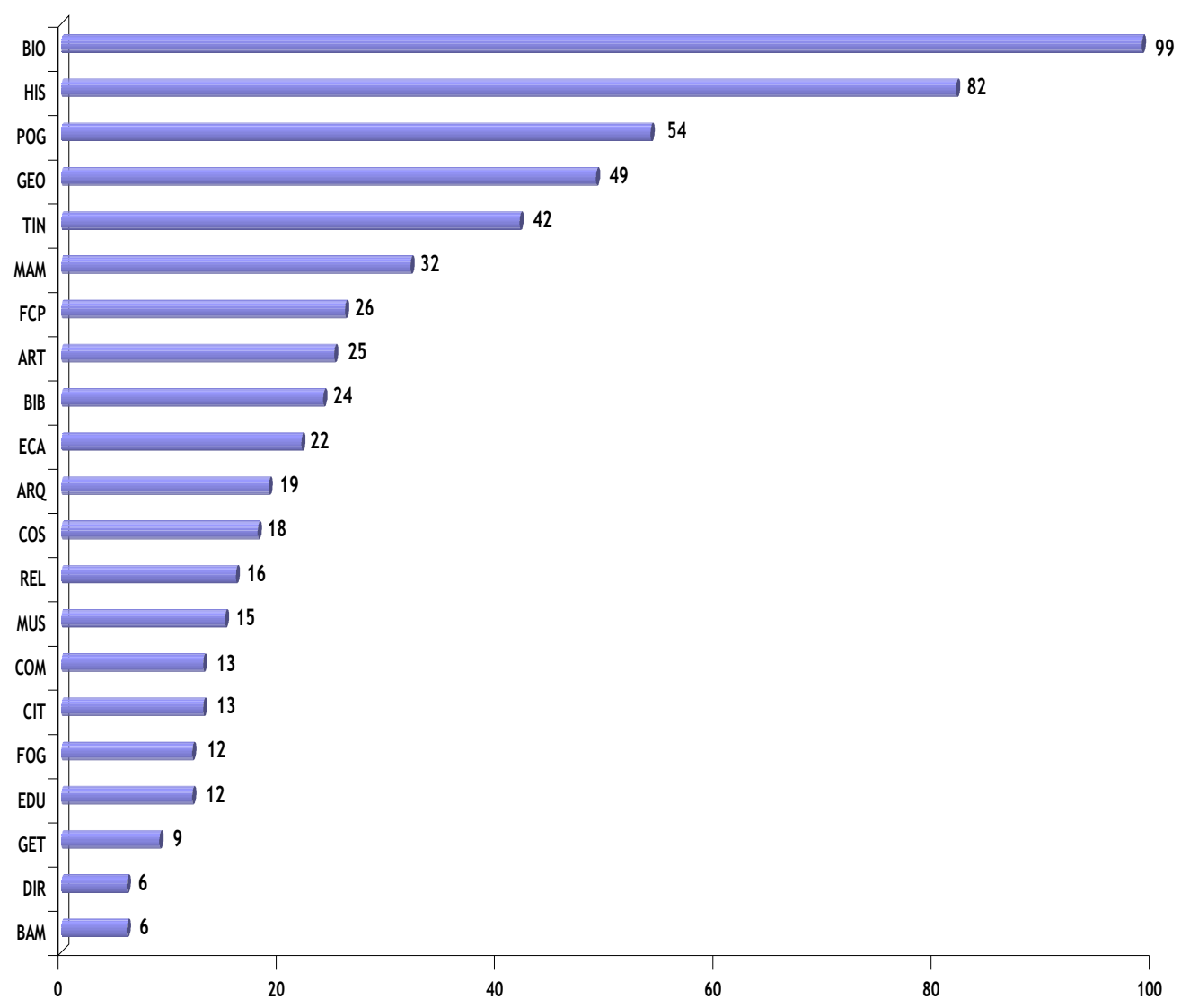

Gráfico 1 - Ranking dos itens bibliográficos por grupos temáticos

A cobertura do estudo limita-se a fontes de referência, 6\%, e outras fontes de informação, 94\%. Na tipologia de obras de referência aparecem apenas dois grupos: bibliografias e fontes gerais. As demais obras encontradas estão classificadas em dezenove assuntos, representando maioria do total de registros.
Do conjunto de bibliotecas visitadas, devidamente escolhidas pela sua representatividade, percebe-se que seis bibliotecas concentram a maior parte do total de registros presentes na listagem, conforme 0 Gráfico 2. 

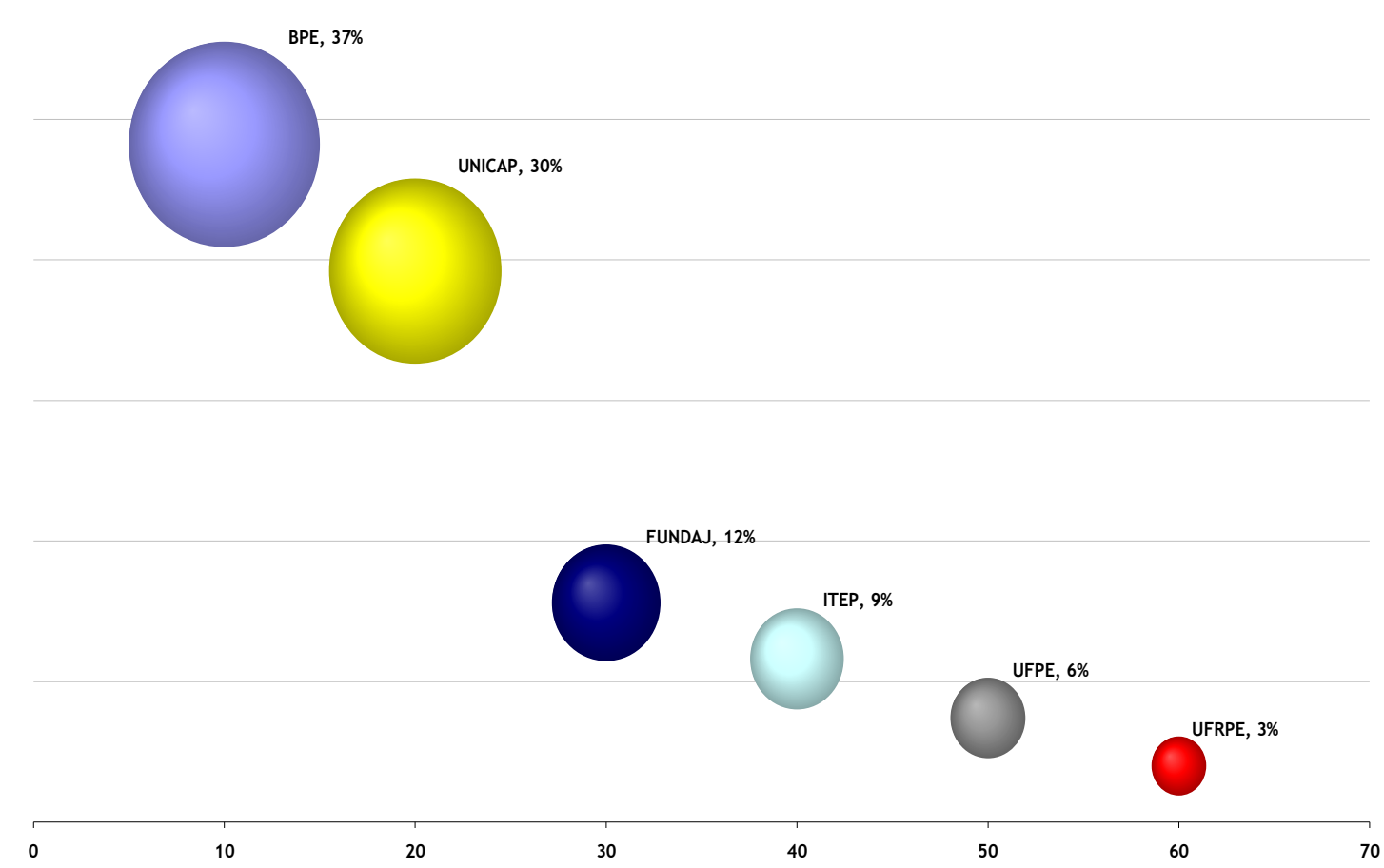

Gráfico 2 - Ranking dos Itens bibliográficos nas bibliotecas

Observa-se que na primeira posição aparece a Biblioteca Pública do Estado de Pernambuco, representada pela Coleção Pernambucana, que tem a função de reunir a produção editorial de autores e editoras pernambucanas. Do grupo acima destacase a presença de três bibliotecas universitárias pertencentes à Universidade Católica de Pernambuco (privada), à Universidade Federal de Pernambuco e à Universidade Federal Rural de Pernambuco (públicas), concentrando $39 \%$ do total de registros. Verifica-se ainda a presença de duas bibliotecas especializadas: da Fundação Joaquim Nabuco (área de Ciências Sociais) e do Instituto Tecnológico de Pernambuco (área de Informação Científica e Tecnológica), com $21 \%$ dos itens coletados.
O Gráfico 2 mostra ainda que o número de itens presentes nas seis bibliotecas contabiliza $97 \%$ da produção editorial localizada. Os $3 \%$ restantes das fontes se concentraram nas sete bibliotecas não presentes no gráfico. Da representação gráfica acima, os registros estão distribuídos em diferentes bibliotecas, indicando um aspecto positivo, considerando a vocação daquelas instituições e seu papel na sociedade pernambucana.

\section{CONSIDERAÇÕES FINAIS}

O projeto motivado pela obra de Ann Hartness (1999), tentou descobrir outras fontes existentes sobre Pernambuco, ampliando então o retrato publicado do estado. O processo de construção dessa bibli- 
ografia envolveu etapas meticulosas e com

grande consumo de tempo desde a coleta

"in-loco", até a elaboração dos índices au-

xiliares para a recuperação das fontes.

Os resultados apresentados, embora parciais, podem ser motivo de análises bibliométricas de variáveis como: autoria, editoras, períodos de tempo, assuntos, locais e os possíveis cruzamentos.

O trabalho ainda incompleto está aberto a novas inclusões e interpretações, para chegar-se a um retrato mais atualizado e concreto da produção editorial do estado, nas áreas concernentes à pesquisa. Isto levando em conta a própria natureza da atividade: inesgotável e sempre a ser completada.

A publicação deverá oferecer informações e servir como indicador para um amplo público, desde profissionais da informação, pesquisadores, estudiosos, técnicos, professores e alunos.

\section{REFERÊNCIAS}

BUFREM, Leilah Santiago (Coord.). Catálogo coletivo de dicionários da língua portuguesa em bibliotecas de Curitiba. Curitiba: Departamento de Biblioteconomia da UFPR, 1993.

CAMPELLO, Bernadete Santos; CENDÓN, Beatriz Valadares; KREMER, Jeannette Marguerite (Org.). Fontes de informação para pesquisadores e profissionais. Belo Horizonte: Ed. da UFMG, 2000.

CUNHA, Murilo Bastos da. Para saber mais: fontes de informação em ciência e tecnologia. Brasília: Briquet de Lemos, 2001.
FIGUEIREDO, Laura M. de; CUNHA, Lélia G. C. da. Curso de bibliografia geral. Rio de Janeiro: Record, 1967.

FIGUEIREDO, Nice Menezes de. A modernidade das cinco leis de Ranganathan. Ciência da Informação, Brasília, v. 21, n. 3 , p. 186-191, set./dez. 1992.

FONSECA, Edson Nery da. Bibliografia de obras de referência pernambucanas. Recife: Imprensa Universitária, 1964.

GROGAN, Denis. A prática do serviço de referência. Brasília: Briquet de Lemos, 1995.

HARTNESS, Ann. Brasil: obras de referência, 1965-1998. Brasília: Briquet de Lemos, 1999.

NORONHA, Daisy Pires. O ensino de bibliografia por módulo. Revista da Escola de Biblioteconomia da UFMG, Belo Horizonte, v. 11, n. 1, p. 53-72, mar. 1982.

\section{Title}

The Pernambuco's world through information sources : 1965-2003.

\section{Abstract}

The article describes a study conducted in order to compile a bibliography of information sources about Pernambuco, from 1965 to 2003. It presents the partial results, related to the amount of items by subject and its location in six libraries from Recife city. The final report might include: bibliographical reference, contents and library notes with retrieval by name and subject index. This bibliography intends to be used both as work and research tool for information professionals, as well as for different types of users. 


\section{Keywords}

Information sources. Bibliography. Pernambuco.

\section{Título}

El mundo pernambucano a través de fuentes de información: 1965-2003.

\section{Resumen}

El artículo describe estudio hecho con fines de compilar una bibliografia de fuentes de información sobre el asunto Pernambuco, en el período de 1965 a 2003. Presenta resultados parciales, relativos a la cantidad de registros por asunto y localización de los mismos en seis bibliotecas de la Ciudad de Recife. El producto final debe incluir: referencia bibliográfica, nota de contenido y biblioteca, con recuperación a través de índices onomástico y de asunto. La bibliografia pretende servir de instrumento de trabajo y investigación, tanto para profesionales de la información, cuanto para diferentes tipos de usuarios.

\section{Palabras Clave}

Fuentes de información. Bibliografías. Pernambuco.

Recebido em: 30.04 .2008

Aceito em: 09.02.2009

\section{AGRADECIMENTOS}

Agradecemos a participação das alunas do Curso de Biblioteconomia da UFPE:

Amanda Carla G. do Nascimento; Amanda Tavares S. Lima; Ana Cristina Vieira; Giseani Bezerra da Silva; Jaqueline Karla C. Marques; Lígia Santos da Silva; Susimery Vila Nova Silva. 